\title{
Implementación del laboratorio de termocronología de baja temperatura: Intercomparación del Servicio Geológico Colombiano - Universidad Grenoble Alpes
}

\section{Implementation of the low-temperature thermocronology laboratory: Intercomparison of the Colombian Geological Survey-Grenoble Alpes University}

\author{
S. Amaya ${ }^{1, a)}$, C. Urueña ${ }^{1}$, M.L.Peña ${ }^{1}$, L. Rayo ${ }^{1} ;$ M. Bernet $^{2}$ \\ ${ }^{1}$ Servicio Geológico Colombiano, Bogotá, Dirección Asuntos Nucleares, Carrera 50 No. 26-20, Bogotá, D.C., Colombia \\ ${ }^{2}$ Insitut des Sciences de la Terre, Université de Grenoble-Alpes, Grenoble, France \\ Autor para correspondencia: ${ }^{\text {a) }}$ samaya@sgc.gov.co
}

Resumen

Este artículo contiene una descripción histórica del proceso de implementación del Laboratorio de Termocronología de baja temperatura en el Servicio Geológico Colombiano; el cual está enfocado en datación termocronológica por el método de huellas de fisión en apatitos y circones. En este trabajo se describe la metodología aplicada, los resultados del proceso de intercalibración con el Laboratorio ISTerre de la Universidad Joseph Fourier e intercomparación interna de los analistas del Laboratorio del SGC, así como los ensayos de viabilidad y condiciones de irradiación para análisis de huellas de fisión Reactor Nuclear IAN-R1, con el fin de dar a conocer a la comunidad científica las capacidades técnicas del laboratorio y la confiabilidad de los resultados obtenidos.

Palabras clave: Apatitos, Circones, Colombia, Datación, Huellas de fisión, Termocronología.

\begin{abstract}
This paper presents a historical description of the implementation process of the Low Temperature Thermochronology laboratory at the Colombian Geological Survey. The laboratory is focused on thermochronological dating of apatites and zircons with the fission track method. Here we explain the applied methodology, the results of the inter-calibration process performed in collaboration with the fission-track laboratory at ISTerre Joseph Fourier University and the internal inter-comparison of the SGC laboratory analysts. Furthermore, a viability study and irradiation conditions for fission track analysis in the nuclear reactor IAN-R1 is presented, in order to demonstrate the technical facilities, precision and reliability of the results obtained in the SGC laboratory to the scientific community.
\end{abstract}

Keywords: Apatites, Zircons, Colombia, Dating, Fission tracks, Thermochronology. 


\section{INTRODUCCIÓN}

El análisis de huellas de fisión en apatitos y circones es una técnica analítica recientemente implementada en Colombia, fundamental en la reconstrucción de la historia termal de las rocas y minerales, que mediante su uso permite limitar un amplio rango de problemas geológicosincluyendo: estudios tectónicos, procedencia, depósitos minerales, evolución de cuencas de carbones e hidrocarburos, estratigrafía volcánica, evolución del paisaje, evolución espacial y temporal de los sistemas de fallas, etc. En el año 2009 se inició la implementación de esta técnica en el Servicio Geológico Colombiano (SGC) realizando ejercicios de intercalibración paralelamente con los laboratorios de la Universidad EAFIT de Medellín y con la Universidad Joseph Fourier (ahora la Universidad Grenoble Alpes) en Francia, logrando después del año 2012 producir información que soporta y hace parte de diferentes proyectos que se han ejecutado y actualmente se desarrollan en el SGC. Son varias las publicaciones nacionales realizadas a partir del 2011 (e.g. [1-7]) y en el segundo semestre del año 2016 se publicó el primer artículo internacional en la revista Journal Volcanology and Geothermal Research y dos más ya fueron sometidos a evaluación (e.g. Amaya et al., en revisión, Lithos [8]; Amaya et al., en revisión, Tectonics [9]).

Este documento presenta la historia de cómo se logró la implementación, adecuación de la infraestructura científica y tecnológica del laboratorio de termocronología del SGC y los resultados obtenidos en ejercicios de intercalibración con la Universidad Joseph Fourier (Francia). Esta información es de interés porque permite mostrar la evolución y el nivel que se ha logrado alcanzar en el laboratorio de termocronología del SGC, dándolo a conocer a la comunidad científica colombiana especialmente en el área de geociencias.

\section{ANTECEDENTES}

La implementación del laboratorio de termocronología del Servicio Geológico Colombiano, requirió elaborar estudios para el diseño del laboratorio, de acuerdo a las etapas necesarias para realizar el análisis de manera autónoma y eficiente de las muestras. El área seleccionada está ubicada en el ala norte del Bloque A de la sede CAN del SGC, en el mismo edificio donde se encuentran las instalaciones del Reactor Nuclear IAN-R1.

Los expertos doctor Matthias Bernet de la Universidad Joseph Fourier de Grenoble (Francia), la doctora Gloria Toro de la Universidad EAFIT junto con personal del SGC evaluaron y dirigieron la adecuación física del laboratorio de huellas de fisión, la realización del diseño y elaboración de planos. Las obras de adecuación del laboratorio se terminaron en primer semestre de 2012 y actualmente cuenta con la infraestructura física y tecnológica finalizada (Figura 1).

Figura 1. Panorámica del laboratorio de Huellas de Fisión, de izquierda a derecha: zona de pulido, ataque químico y Separador Magnético Frantz

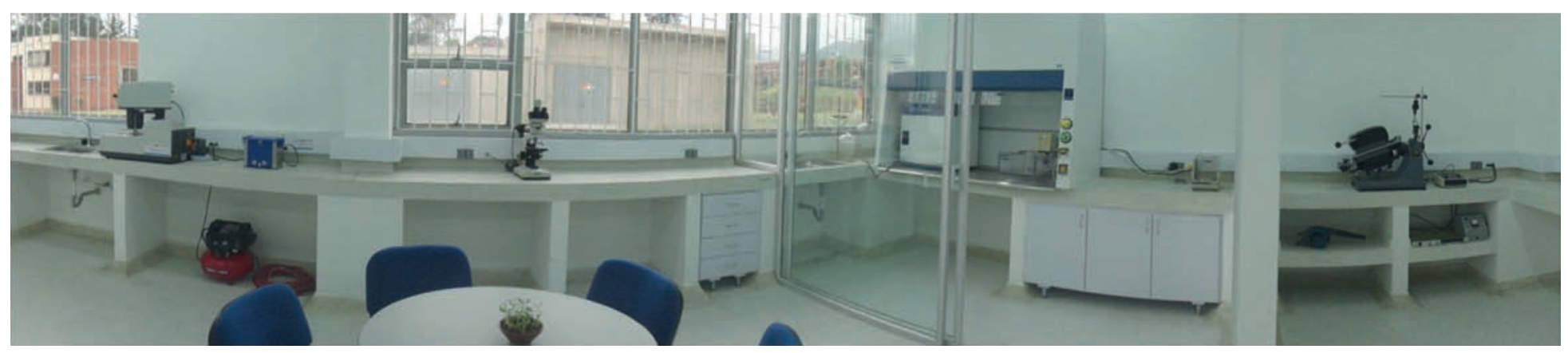


La distribución de las áreas se diseñó buscando tener un proceso secuencial del montaje de las muestras. El laboratorio cuenta con área de separación y almacén de muestras, es el lugar donde se realiza la separación por densidad de minerales. La separación magnética mediante el separador de susceptibilidad magnética Frantz ${ }^{\circledR}$, se hace sobre un mesón reforzado apto para soportar el peso y las vibraciones del equipo. En el área de montaje se seleccionan los apatitos y circones fijándolos en resina epóxica y teflón respectivamente. Para hacer estos montajes se cuenta con un estéreo microscopio Olympus SZ7X adaptado a un sistema de luz LED, una plancha caliente Cimarec de $26.7 \mathrm{x}$ $26.7 \mathrm{~cm}$ y otros insumos.

Para el proceso de pulido de los montajes se cuenta con una pulidora con cabezal automático marca MEKTON (Forcipol 1V/Forcimat), con suministro de agua y aire comprimido. Un baño ultrasonido ELMA E30 para limpieza de los montajes y un microscopio petrográfico NIKON Labophot-Pol con sistema de luz reflejada para realizar el control de calidad del proceso.

La zona de ataque químico de los apatitos y circones cuenta con una cabina extractora de vapores y gases. Mediante el ataque se revelan las huellas de fisión espontáneas tanto de apatitos como de circones. La cabina extractora es necesaria por razones de seguridad debido a la naturaleza de los reactivos químicos utilizados en estos procesos. En el interior de la cabina se ubican el horno ESCO OFA, el baño termostatado LAUDA E-100 y fuera de la cabina se ubica la balanza electrónica.

En el área de conteo y análisis de huellas de fisión se tienen dos Microscopios Olympus BX51-P con accesorios, acoplados a la platina motorizada Kinetek y al sistema de conteo de huellas de fisión FTStage, controlados por un computador (Figura 2).

Adicionalmente, el SGC cuenta con el Reactor Nuclear IAN-R1, esta instalación tipo TRIGA (Training, Research, Isotopes, General Atomics) creada para la investigación y el entrenamiento en técnicas nucleares, única en el país, tiene dentro de sus posibles usos la irradiación de materiales geológicos tanto para análisis de activación neutrónica como para datación por huellas de fisión. Por esta razón, durante los años 2010 y 2012 se realizaron ensayos con el fin de analizar la viabilidad y aportar información para la determinación de las condiciones de irradiación de muestras geológicas para datación con el método de huellas de fisión. Para ello se realizaron irradiaciones de monitores de fluencia (metálicos y vidrios dosimétricos) y muestras estándares de la toba de Fish Canyon.

Figura 2. Sistema FT Stage para el conteo de huellas de fisión del Servicio Geológico Colombiano

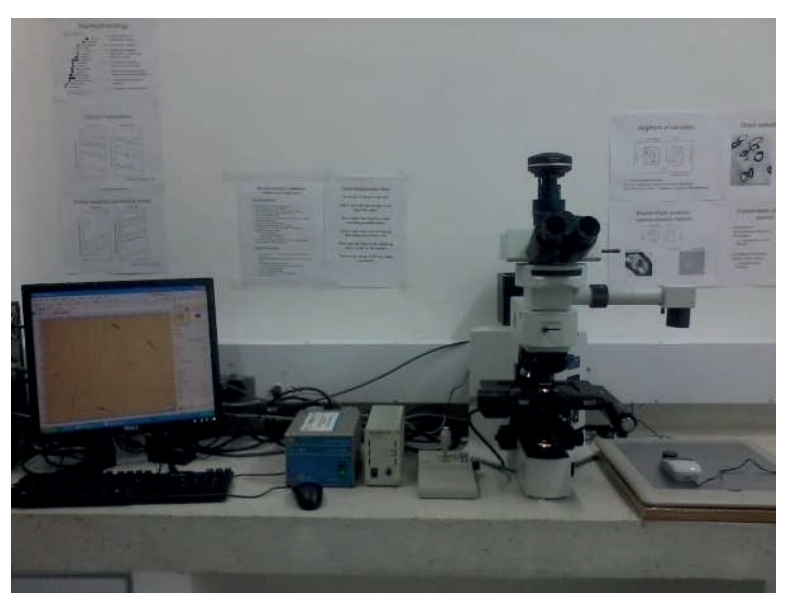

\section{DESCRIPCIÓN DEL MÉTODO}

El método analítico del detector externo en huellas de fisión en apatitos y circones (termocronómetros de baja temperatura), se basa en el principio de fisión espontánea del ${ }^{238} \mathrm{U}$. El Uranio es un elemento que ingresa con facilidad en la estructura cristalina de apatitos, circones, titanita, entre otros minerales; en concentraciones que varían desde 50 a $\sim 1000 \mathrm{ppm}$. La fisión espontánea consiste en la división del ${ }^{238} \mathrm{U}$ en dos mitades, por ionización y desplazamiento electrostático (Figura 3), moviéndose independientemente a grandes velocidades, deteniéndose solo tras causar grandes daños a las estructuras que se encuentran en su trayectoria [10]. 
Figura 3. Se muestra cómo en una red cristalina se produce ionización y desplazamiento electrostático como producto de la fisión espontánea del $2^{38} \mathrm{U}$. Tomado de Fleischer et al.[10]

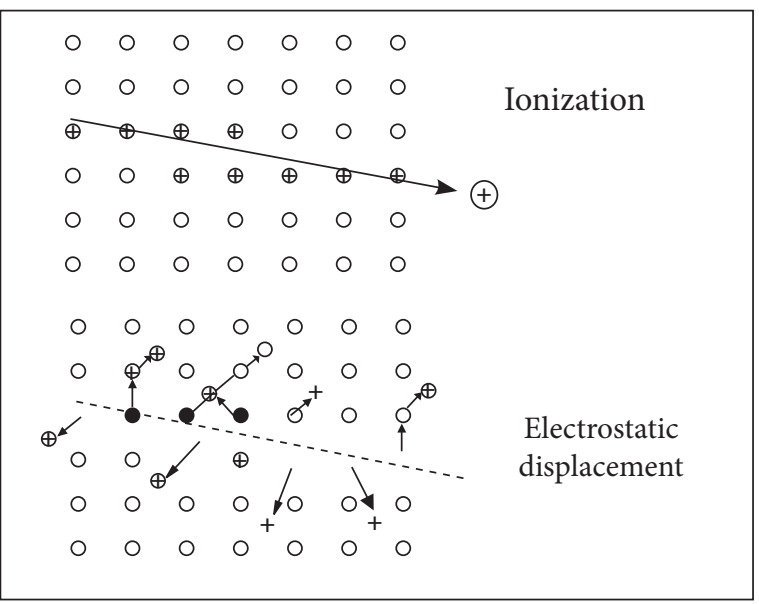

El daño en la estructura se registra en forma de trayectorias llamadas huellas de fisión ([10]; Figura 4), dichas huellas se cuentan usando un microscopio óptico después de haber atacado químicamente la superficie, previamente pulida (Figura 5.)

Figura 4. Daño en la estructura cristalina o huella de fisión ocasionado por la fisión espontánea del ${ }^{238} \mathrm{U}$. Las huellas de fisión del ${ }^{238} \mathrm{U}$. Tomado de Fleischer et al. [10]

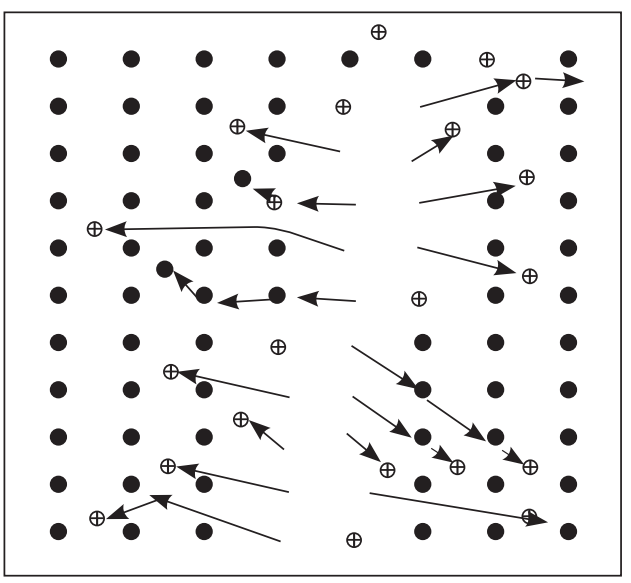

Figura 5. Huellas de fisión en circón. Las huellas están orientadas principalmente al azar. Foto tomada en el laboratorio de termocronología del Servicio Geológico Colombiano.

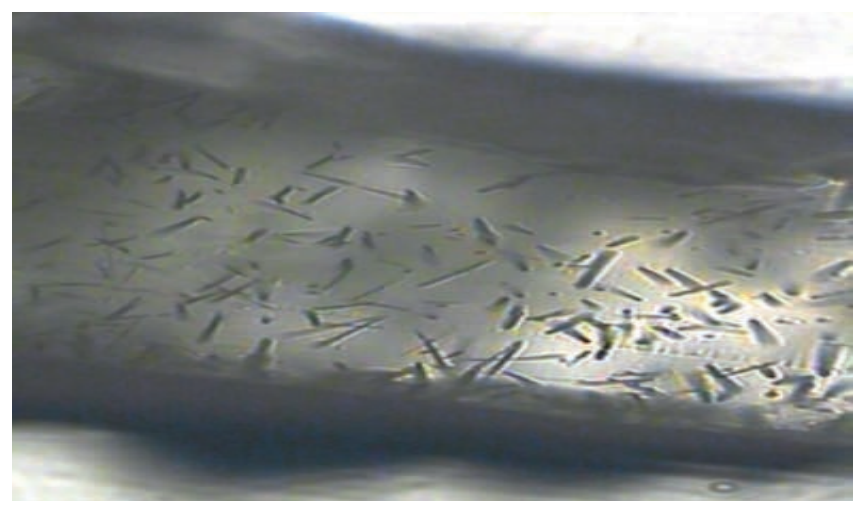

La cantidad de $\mathrm{U}$ (total) se determina por la densidad de huellas espontáneas que hay en un cristal y mediante el recuento de un segundo grupo de huellas creadas por fisión inducida de los átomos de ${ }^{235} \mathrm{U}$, provocada artificialmente en un reactor nuclear.

Conociendo la tasa de fisión de fisión del ${ }^{238} \mathrm{U}\left(\mathrm{t}_{1 / 2}\right.$ del ${ }^{238} \mathrm{U} \approx 8,2 \times 10^{15}$, años, [11]) se llega a una fecha al comparar el número de huellas producidas espontáneamente con la cantidad de ${ }^{238} \mathrm{U}$ de la muestra.

En principio, el cálculo de la edad por huellas de fisión se basa en la determinación del número de huellas espontáneas y la determinación del número de átomos de ${ }^{238} \mathrm{U}$ por unidad de volumen en la muestra [12]. La irradiación de las muestras con un flujo de neutrones termalizado, induce la fisión de ${ }^{235} \mathrm{U}$ y genera huellas inducidas; el número de huellas inducidas indica la abundancia de ${ }^{235} \mathrm{U} y$, como la abundancia relativa de isótopos de uranio es relativamente constante en la naturaleza, a partir de la relación ${ }^{235} \mathrm{U} /{ }^{238} \mathrm{U}$ se puede estimar la abundancia de ${ }^{238} U[12,13]$. En conclusión, el conteo del número de huellas inducidas permite estimar la concentración de uranio o padre en el cristal y, en el conteo del número de huellas espontáneas se estima la concentración del producto hijo en el cristal [14]. De acuerdo con lo anterior, la ecuación fundamental de edad es la siguiente: 


$$
t=\frac{1}{\lambda_{D}} \operatorname{Ln}\left[\left(\frac{\lambda_{D}}{\lambda_{f}}\right)\left(\frac{\rho_{S}}{\rho_{i}}\right) I \varphi \sigma+1\right]
$$

donde $\lambda_{\mathrm{D}}$ es la constante de decaimiento radiactivo del ${ }^{238} \mathrm{U}$; $\lambda_{\mathrm{f}}$ es la constante de decaimiento por fisión del ${ }^{238} \mathrm{U}$; $\varphi$ es el flujo de neutrones termalizado; $I$ es la razón isotópica ${ }^{235} \mathrm{U} /{ }^{238} \mathrm{U}$; $\sigma$ es la tasa de fisión inducida de átomos de ${ }^{235} \mathrm{U}$ debida al flujo de neutrones y $p_{s} / p_{i}$ es la relación entre la cantidad de huellas fósiles e inducidas en la irradiación, respectivamente. Los valores constantes de $\lambda_{\mathrm{D}}\left(1,55125 \times 10^{-10}\right.$ año $\left.^{-1}\right), I\left(7,2527 \times 10^{-3}\right)$ y $\sigma(580,2$ $\left.\mathrm{x} 10^{-24} \mathrm{~cm}^{2}\right)$ son aceptados ampliamente, sin embargo se requiere evaluar las densidades de huellas $\left(\rho_{\mathrm{s}}\right.$ y $\left.\rho_{\mathrm{i}}\right)$, la constante de decaimiento por fisión $\operatorname{del}{ }^{238} \mathrm{U}\left(\lambda_{\mathrm{f}}\right)$ y la fluencia de neutrones termalizados $(\varphi)$ para determinar la edad [15].

Para solucionar los problemas para la determinación de la fluencia absoluta de la irradiación y de la definición de la constante de fisión del ${ }^{238} \mathrm{U}$ [15], formularon el método del detector externo. Este método de medición relativa, emplea muestras con edades estándares y monitores de fluencia (vidrios dopados con U) para calibrar la fluencia neutrónica, esta relación es conocida

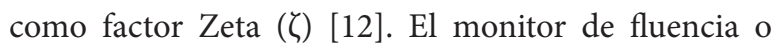
vidrio dosimétrico provee una medida relativa de la fluencia neutrónica y es el enlace con la edad estándar en la ecuación de la edad. El factor de calibración zeta

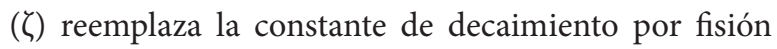
del $238 \mathrm{U}(\lambda \mathrm{f})$, la razón isotópica ${ }^{235} \mathrm{U} /{ }^{238} \mathrm{U}$ (I) y la tasa de fisión inducida de átomos de ${ }^{235} \mathrm{U}$ debida al flujo de neutrones $(\sigma)$, por medio de la relación:

$$
\zeta=\frac{\varphi \sigma I}{\lambda f}
$$

En la práctica el factor $\zeta$ es evaluado por análisis repetidos de una serie de edades estándares, se recomiendan al menos dos montajes por cada fase mineral y que hayan sido incluidos en diferentes irradiaciones. Se utiliza la siguiente ecuación para obtener $\zeta$ :

$$
\zeta=\frac{e^{\left(\lambda_{D} t_{s t d}\right)}-1}{\lambda_{D}\left(\frac{\rho_{s}}{\rho_{i}}\right)_{(s t d)} \rho_{d}}
$$

donde, $\lambda \mathrm{D}$ es la constante de decaimiento radiactivo del ${ }^{238} \mathrm{U}$; tstd es la edad del estándar (determinada por otro método como K/Ar o $\left.{ }^{39} \mathrm{Ar}-4^{0} \mathrm{Ar}\right)$; $(\rho s / \rho \mathrm{i})_{\text {std }}$ es la razón entre las densidades de huellas fósiles e inducidas en el estándar, respectivamente; y $\rho_{d}$ es la densidad de huellas inducidas por un vidrio dosimétrico (vidrio con un contenido conocido de $\mathrm{U}$ ) sobre un detector externo (mica) durante la irradiación del estándar.

Por tanto la ecuación de la edad recomendada por [16] para la datación por huellas de fisión, al sustituir el factor $\zeta$ en la ecuación inicial, es:

$$
t=\frac{1}{\lambda_{D}} \operatorname{Ln}\left[1+\lambda_{D} \zeta\left(\frac{\rho_{s}}{\rho_{i}}\right) \rho_{d}\right]
$$

El reloj se pone a cero cuando el mineral o cristal alcanza la temperatura de cierre que para el circón está en un rango de $180^{\circ}$ a $260^{\circ}$ centígrados y el apatito entre $80^{\circ}$ y $120^{\circ}$ centígrados (Figura 6 ). La temperatura de cierre se refiere a la temperatura a la cual el sistema no tiene más pérdidas de los productos (Isótopos hijos o huellas de fisión) durante el enfriamiento, esta depende de varios factores que afectan según sea el sistema termocronométrico. Los sistemas de huellas de fisión en circones y apatitos (ZFT, AFT) se ven afectados por la velocidad de enfriamiento ([10, 17]; Figura 6); la composición química afecta a las huellas de fisión en apatitos (AFT) y el daño por radiación a las huellas de fisión en circones (ZFT) [12].

Figura 6. Se muestra la relación de la temperatura de cierre vs velocidad de enfriamiento para los diferentes sistemas termocronométricos. Tomado de [17]

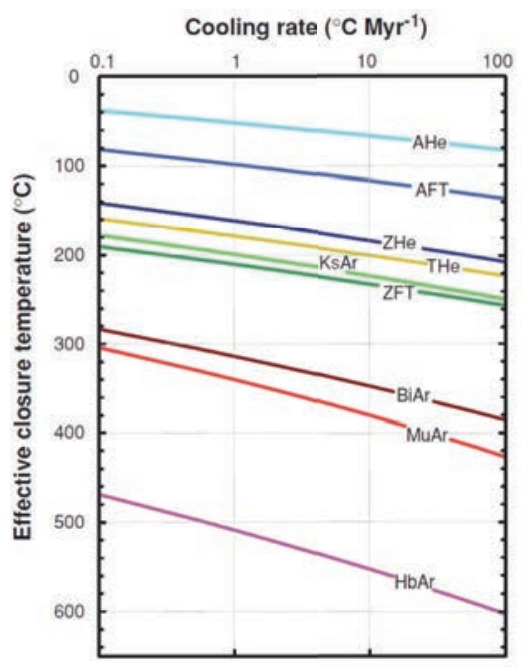

En el Laboratorio de Termocronología del SGC se aplica el método del detector externo (Figura 7), en 
el cual las huellas espontáneas son contadas en un área seleccionada del mineral después ser atacado químicamente, mientras las huellas inducidas son contadas en la misma área de un detector externo (mica) con bajo contenido de uranio, que es adicionado a la muestra antes de la irradiación y después del enfriamiento radiactivo se ataca químicamente. De este modo, las huellas espontáneas e inducidas son contadas en la misma superficie plana de cada cristal y la distribución heterogénea de uranio dentro y entre los cristales es despreciable [15].

Figura 7. Esquema del procedimiento que se sigue con el método del detector externo.

Tomado de [14].

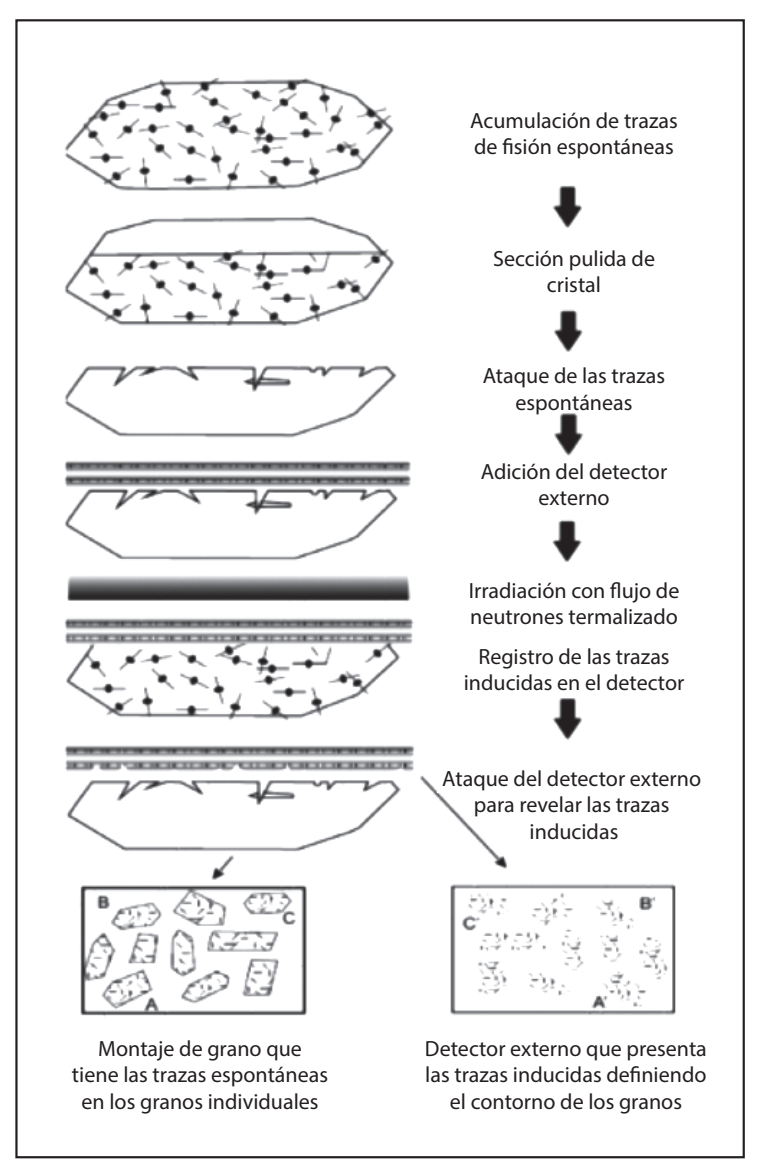

\section{RESULTADOS}

Intercalibración Laboratorio ISTerre de la Universidad Joseph Fourier e intercomparación interna de los analistas del Laboratorio del SGC.
Los ejercicios de intercalibración realizados durante los años 2010 y 2011 consistieron en realizar el conteo de huellas de fisión en muestras estándares para obtener valores de fluencia y el factor Zeta $(\zeta)$ de diferentes irradiaciones, por parte de varios analistas en los laboratorios de huellas de fisión de las universidades EAFIT, Joseph Fourier y en el SGC ([1]; [18]). Posteriormente nuevos analistas en el SGC realizaron el ejercicio de conteo y obtención del factor Zeta $(\zeta)$, los valores obtenidos son comparados entre sí y evaluados con respecto a los resultados de la intercalibración con el Laboratorio del ISTerre de la Universidad de Joseph Fourier.

Un ejercicio de intercomparación fue realizado por dos analistas en los Laboratorios del Servicio Geológico Colombiano y la Universidad de Grenoble - Alpes de Francia, con el objetivo de determinar los valores de fluencia de cinco (5) irradiaciones realizadas en el reactor de Múnich (Tabla 1), a partir de los vidrios dosimétricos de referencia (CN1, CN2, CN5, IRMM540R, IRMM-541). En la figura 8 se puede ver que los valores de fluencia obtenidas en los dos laboratorios tienen una factor de correlación R2 $=0.9977$, lo cual muestra el nivel alcanzado por los analistas del Servicio Geológico Colombiano en la técnica de huellas de fisión.

Para la determinación del factor Zeta $(\zeta)$, es necesario realizar inicialmente el conteo de huellas inducidas en el vidrio dosimétrico sobre el detector externo, a fin de determinar el termino $\rho d$, que representa la fluencia o capacidad de observación del analista. El parámetro de fluencia varía para cada analista y se da en respuesta directa del flujo de neutrones termalizados $(\varphi)$ con el que fue irradiado el grupo de muestras y el contenido de Uranio en el vidrio dosimétrico de referencia ( $\mathrm{CN} 1$, CN2, CN5, IRMM-540R, IRMM-541).

Los valores de zeta para apatitos y circones obtenidos en diferentes irradiaciones y estándares por los analistas en el laboratorio de SGC se relacionan en las Tablas 2 y 3; las Figuras 9 y 10 muestran el valor promedio de zeta de los analistas con respecto a la referencia de intercalibración, así mismo se incluyen los valores reportados por otros autores $([13,19])$ de acuerdo al vidrio dosimétrico usado, los cuales representan el rango entre el cual es aceptado el valor de zeta para la ejecución de análisis de huellas de fisión. 
En el caso de apatitos (Figura 9) el valor de zeta esperado de acuerdo al vidrio de referencia usado en el laboratorio del SGC es aproximadamente a 300; para circones (Figura 10) el valor esperado es cercano a 150 y menor a 200 .

Desde su implementación, el Laboratorio de Termocronología del SGC ha participado evaluando la evolución térmica de carbones en la ejecución de proyectos como: Gas Asociado a los Carbones de la Formación Guaduas en el Sinclinal de Úmbita, Boyacá; Gas Asociado a los Carbones de la Formación Umir en el Sinclinal de Andes, en la Cuenca del Valle Medio del Magdalena; Gas Asociado a los Carbones de la Formación Umir en el Sinclinal de Armas, en la Cuenca del Valle Medio del Magdalena, el cual ha contribuido a que en Colombia se haya enfocado a la exploración de hidrocarburos no convencionales hacia el Gas Asociado a Carbones (CBM, tomado de las siglas en ingles Coal Bed Methane), como importantes objetivos en las cuencas carboníferas en el territorio colombiano.

Figura 8. Factor de correlación R2 ejercicio de intercomparación Laboratorios de termocronología SGC -Universidad Grenoble Alpes

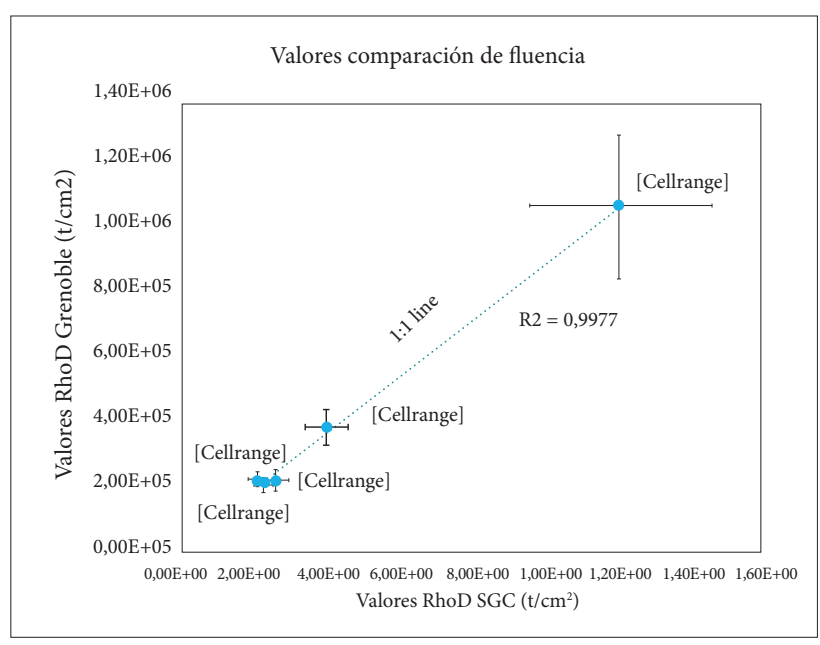

Tabla 1. Valores de fluencia a partir de vidrios dosimétricos de referencia en el ejercicio de intercomparación laboratorios SGC - Universidad Grenoble Alpes. Comparación valores de fluencia SGC Universidad Grenoble-Alpes

\begin{tabular}{c|c|c|c|c}
\hline \multirow{2}{*}{} & \multicolumn{2}{|c|}{ SGC } & \multicolumn{2}{c}{ Universidad Grenoble Alpes } \\
\cline { 2 - 5 } & Rhod & 2 2SE Rhod & Rhod & 2 SE Rhod \\
\hline Irr 18-2012 & $2,35 \times 10^{5}$ & $2,11 \times 10^{4}$ & $2,10 \times 10^{5}$ & $2,52 \times 10^{4}$ \\
\hline Irr 16-2013 & $4,09 \times 10^{5}$ & $5,27 \times 10^{4}$ & $3,87 \times 10^{5}$ & $2,57 \times 10^{4}$ \\
\hline Irr 14-2013 & $1,22 \times 10^{6}$ & $2,50 \times 10^{5}$ & $1,08 \times 10^{6}$ & $3,33 \times 10^{4}$ \\
\hline Irr 1-2010 & $2,72 \times 10^{5}$ & $3,14 \times 10^{4}$ & $2,23 \times 10^{5}$ & $2,19 \times 10^{4}$ \\
\hline Irr 8-2012 & $2,24 \times 10^{5}$ & $2,59 \times 10^{4}$ & $2,23 \times 10^{5}$ & 2 \\
\hline
\end{tabular}


Tabla 2. Factor Zeta para apatitos (Media y 2SE: desviación estándar), determinado en cada estándar de la respectiva irradiación, comparativo para 5 analistas, valores de referencia de intercalibración y valor promedio.

\begin{tabular}{|c|c|c|c|c|c|c|c|c|c|c|c|c|}
\hline Analista & \multicolumn{2}{|c|}{ Ref. intercalibración } & \multicolumn{2}{|c|}{1} & \multicolumn{2}{|c|}{2} & \multicolumn{2}{|c|}{3} & \multicolumn{2}{|c|}{4} & \multicolumn{2}{|c|}{5} \\
\hline Muestra & Media & 2SE & Media & 2SE & Media & $2 \mathrm{SE}$ & Media & 2SE & Media & $2 \mathrm{SE}$ & Media & 2SE \\
\hline Muestra & Ref. intercalibración & & 1 & & 2 & & 3 & & 4 & & 5 & \\
\hline DUR-3_3-2010 & 338,02 & 28,2 & & & & & & & & & & \\
\hline DUR-1_7-2011 & & & & & 257,23 & 32,5 & 240,54 & 19,5 & 258,83 & 27,6 & 314,76 & 27,8 \\
\hline DUR-2_7-2011 & & & & & 215,18 & 33,5 & 248,57 & 22,7 & 230,34 & 25,2 & & \\
\hline FCT-3_7-2011 & & & & & 280,18 & 44 & 252,93 & 36,2 & 248,65 & 44,5 & 263,24 & 42,1 \\
\hline FCT-4_7-2011 & 241,57 & 37 & & & 226,52 & 38,2 & 238,01 & 33,9 & 309,59 & 37,9 & 286,36 & 45 \\
\hline FCT_8-2012 & & & & & & & & & 280,31 & 19,8 & & \\
\hline DUR-1_13-2012 & & & 249,34 & 46,4 & 192,04 & 20,2 & 290,09 & 30,4 & & & & \\
\hline DUR-A_8-2013 & & & 242,74 & 45,6 & 270,45 & 18,8 & & & & & & \\
\hline DUR-B_8-2013 & & & 216,22 & 41,8 & 285,62 & 22 & & & & & & \\
\hline DUR-E_14-2013 & & & 343,65 & 33,5 & & & & & 233,47 & 28,1 & & \\
\hline FCT-C_14-2013 & & & 214,37 & 15,6 & & & & & 220,43 & 23,5 & & \\
\hline DUR-1_15-2013 & 303,53 & 9,9 & 203,69 & 45,6 & 315,13 & 24 & & & 264,5 & 15,4 & & \\
\hline DUR-2_15-2013 & & & 210,2 & 30,6 & & & & & 248,32 & 25,7 & & \\
\hline DUR-C_10-2014 & & & 212,09 & 30,7 & 324,97 & 26 & & & & & & \\
\hline DUR-E_9-2015 & & & 238,83 & 22,6 & 325,73 & 20,7 & & & & & & \\
\hline PROMEDIO & 294,37 & 25,0 & 236,79 & 34,7 & 304,38 & 22,3 & 254,03 & 28,5 & 254,94 & 27,5 & 288,12 & 38,3 \\
\hline
\end{tabular}

Tabla 3. Factor Zeta para circones (Media y 2SE: desviación estándar), determinado en cada estándar de la respectiva irradiación, comparativo para 6 analistas, valores de referencia de intercalibración y valor promedio.

\begin{tabular}{|c|c|c|c|c|c|c|c|c|c|c|c|c|c|c|}
\hline Analista & interca & ración & & & & & & & & & & & & \\
\hline Muestra & Media & $2 \mathrm{SE}$ & Media & 2SE & Media & 2SE & Media & $2 \mathrm{SE}$ & Media & 2SE & Media & 2SE & Media & $2 \mathrm{SE}$ \\
\hline BLK-1_1-2010 & 182,18 & 18,1 & 114,17 & 10,3 & 171,63 & 32,8 & 134,36 & 16,3 & & & & & & \\
\hline FCT-1_1-2010 & & & 115,34 & 11,1 & 179,58 & 21,5 & 197,08 & 12,6 & & & & & & \\
\hline FCT-A_8-2012 & 199,81 & 16,4 & 181,49 & 15,4 & 247,65 & 18,5 & & & 146,58 & 17,3 & 280,31 & 20,4 & & \\
\hline FCT-2_8-2012 & & & 200,36 & 14,3 & 216,73 & 16,1 & 228,03 & 12,4 & 172,33 & 12,9 & 150,64 & 10,7 & & \\
\hline FCT-1_18-2012 & & & & & & & 206,78 & 13,8 & & & 157,57 & 18,9 & 198,13 & 12,7 \\
\hline BUL-A_18-2012 & & & & & & & 154,86 & 10,8 & & & 156,72 & 18,2 & 212,05 & 23,4 \\
\hline FCT-3_18-2012 & & & & & & & & & & & 178,18 & 19,3 & 183,03 & 11,3 \\
\hline PROMEDIO & 191,00 & 17,3 & 152,84 & 12,8 & 203,90 & 22,2 & 184,22 & 13,2 & 159,46 & 15,1 & 184,68 & 17,5 & 197,74 & 15,8 \\
\hline
\end{tabular}


Figura 9. Resultados del cálculo del factor Zeta promedio a partir del conteo de muestras estándares de apatitos para diferentes irradiaciones para 5 analistas en el laboratorio del SGC y comparación con el valor de referencia del ejercicio de intercalibración y los valores obtenidos en estudios de estandarización de acuerdo al vidrio dosimétrico empleado

\section{Factor Zeta Apatito}

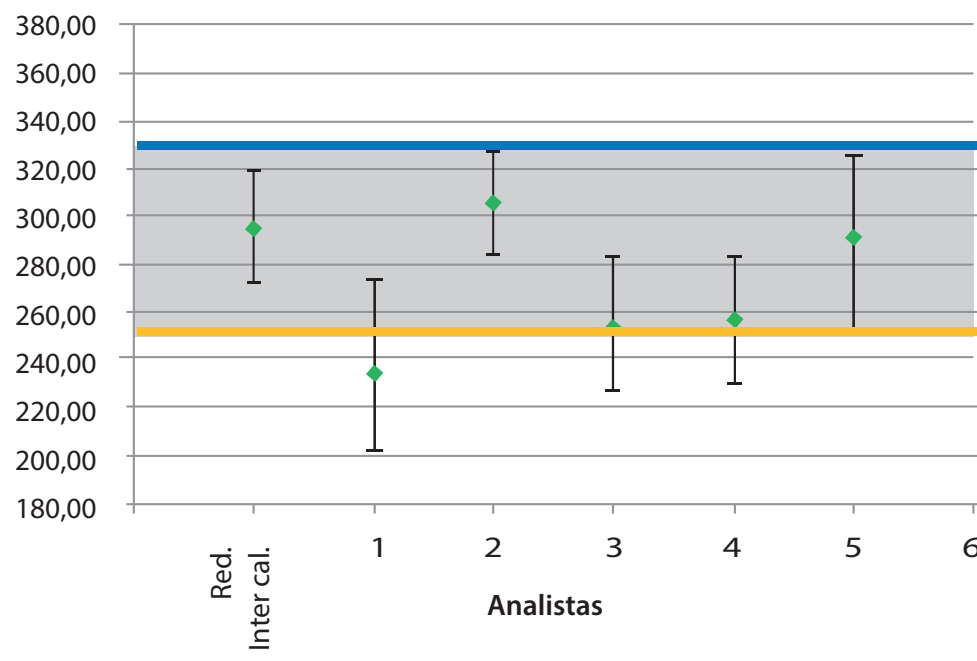

Calibración $\zeta$ con SRM612 (Wagner \& van den Haute, 1992)

- Factor Zeta

Calibración $\zeta$ con IRM-540R (Ghosh, et al., 2016)

Figura 10. Resultados del cálculo del factor Zeta promedio a partir del conteo de muestras estándares de circones para diferentes irradiaciones para 6 analistas en el laboratorio del SGC y comparación con el valor de referencia del ejercicio de intercalibración y los valores obtenidos en estudios de estandarización de acuerdo al vidrio dosimétrico empleado y valor esperado

\section{Factor Zeta Circón}

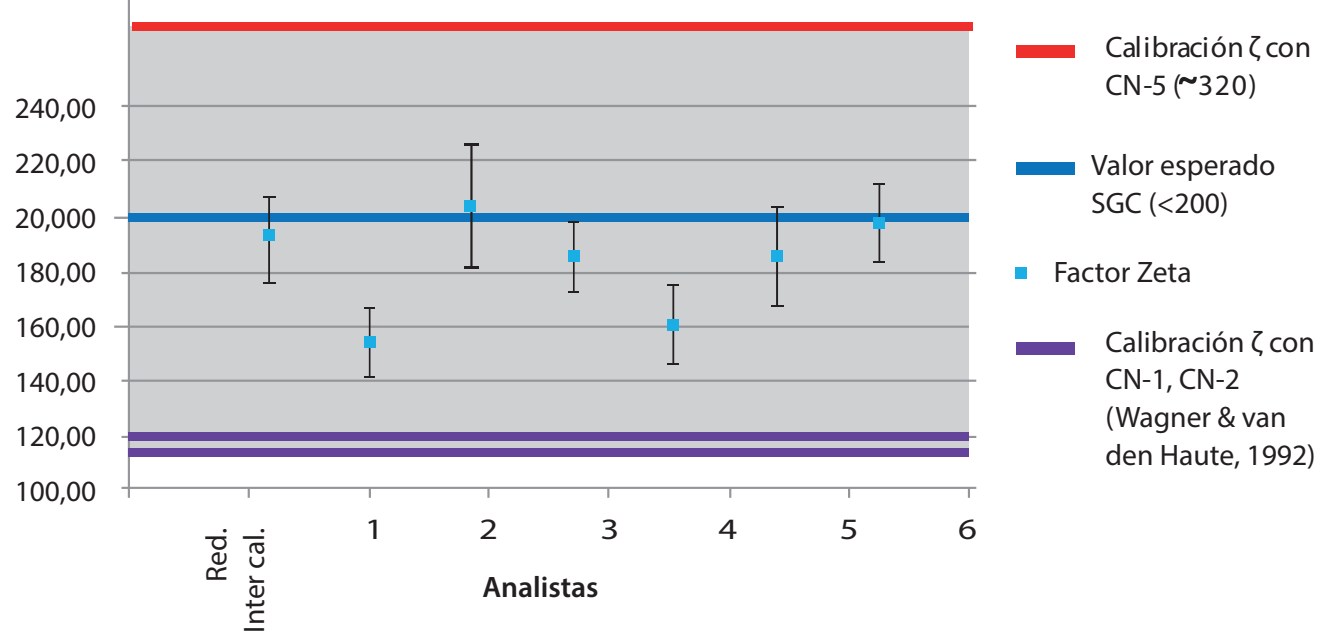


Ensayos de viabilidad y condiciones de irradiación para análisis de huellas de fisión Reactor Nuclear IAN-R1.

Dado que las condiciones de flujo neutrónico y las posiciones de irradiación no son constantes en todas las instalaciones nucleares, es necesario caracterizar detalladamente cada reactor para identificar la posición ideal para irradiar muestras para análisis por huellas de fisión. Para escoger las instalaciones nucleares para estos análisis, se consideran como factores fundamentales [20]: que las fluencias de neutrones térmicos requeridas

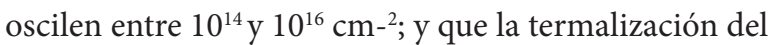
flujo neutrónico sea adecuada para hacer despreciable la contribución de las huellas de fisión inducidas por el ${ }^{232} \mathrm{Th}$ en la densidad de huellas, dado que la relación $\mathrm{Th} / \mathrm{U}$ exhibe variaciones significativas entre muestras en la naturaleza.

Teniendo en cuenta lo anterior, con el objetivo de evaluar las condiciones necesarias para la irradiación de muestras para análisis de huellas de fisión, en coordinación con los integrantes del Laboratorio de Activación Neutrónica y del Reactor Nuclear del SGC, se llegó a la conclusión de la pertinencia de medir el flujo de neutrones térmicos e intermedios en las posiciones F1 y F4 y en la vecindad de la fila G del Reactor Nuclear IAN-R1. Se realizaron mediciones de la actividad de monitores metálicos y vidrios dosimétricos irradiados en el reactor, mediante la técnica de espectrometría de rayos gamma, usando un detector de germanio híper-puro GeHp Camberra CG-1020 acoplado al módulo Inspector y el software Genie-2000 V. 3.2 del Laboratorio de Activación Neutrónica del SGC. Los montajes consistieron en monitores de oro, desnudos $\mathrm{y}$ cubiertos con cadmio, vidrios dosimétricos y muestras estándares de la toba de Fish Canyon para determinación de fluencias y factor Zeta. Los resultados obtenidos permitieron concluir que el flujo térmico, en las posiciones estudiadas, son del orden de 1010 a $10^{11} \mathrm{n} / \mathrm{cm}^{2 \mathrm{~s}}$ y razones de cadmio del orden de las decenas. Las mediciones de densidad de huellas $(\rho \mathrm{d})$ inducidas en las micas de los vidrios dosimétricos (Figura 11), permitieron observar que las posiciones en los alrededores de la fila $G$ del reactor presenta una mayor densidad de huellas que las posiciones $\mathrm{F}$, sugiriendo una mayor fluencia en esta irradiación, consistente con las mediciones de flujo realizadas con los monitores metálicos y la duración de la irradiación.

Figura 11. Microfotografías tomadas en luz polarizada plana transmitida y reflejada de las micas (detectores externos) adheridas a los monitores de flujo neutrónico IRMM-541, en las diferentes posiciones del Reactor Nuclear IAN-R1. En la parte baja cristal de circón del Fish Canyon Tuff (estándar) con las huellas espontáneas (a la derecha) y su respectiva imagen especular en la mica con las huellas inducidas en la irradiación en la posición G3-G4 (izquierda), nótese la gran densidad de huellas en esta muestra. La barra al extremo derecho indica 10 micras
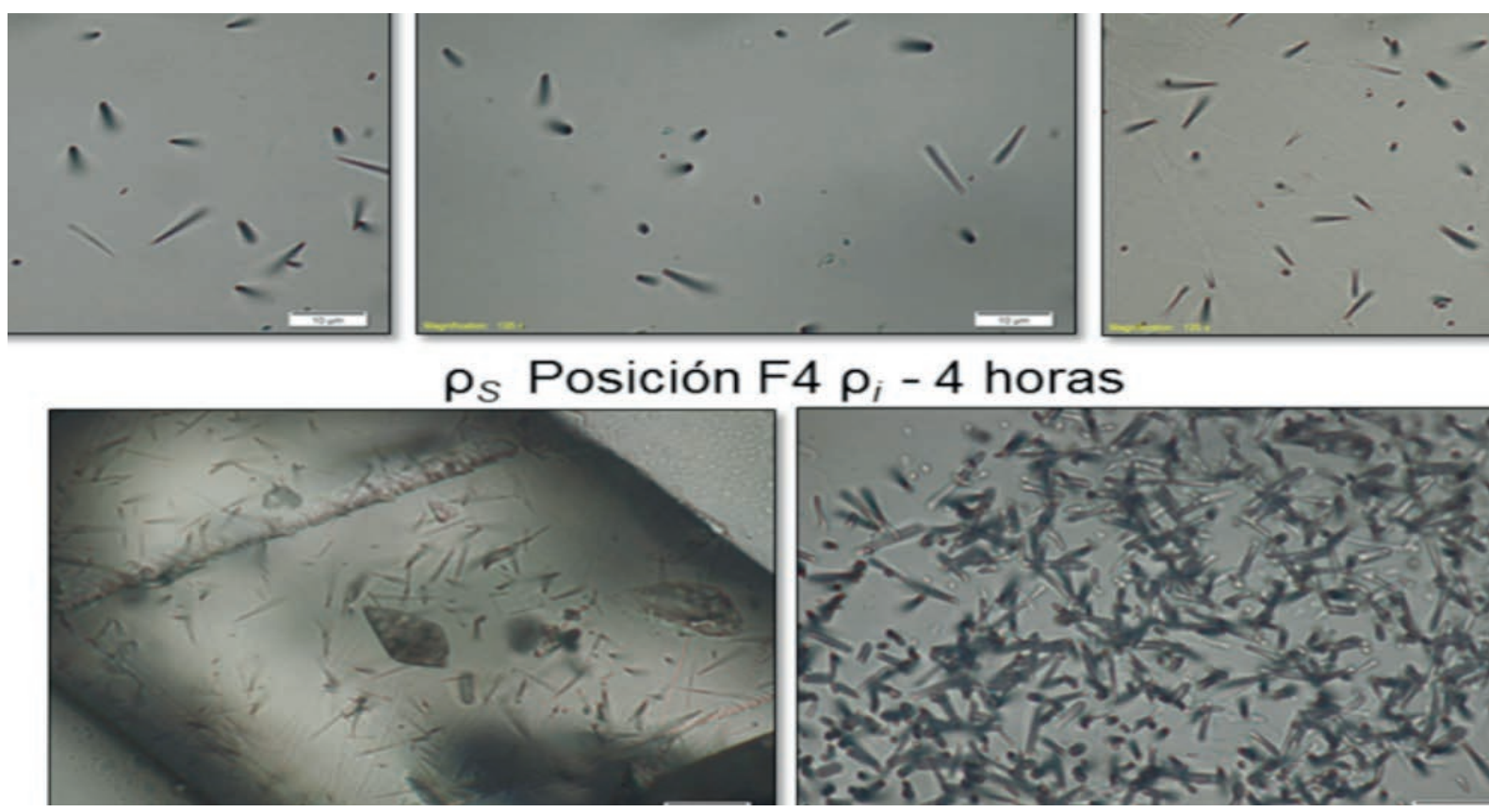


\section{CONCLUSIONES}

Los resultados obtenidos en la evaluación de la evolución térmica de los carbones en los diferentes proyectos ejecutados por el SGC enfocados a la exploración de los hidrocarburos no convencionales hacia los CBM, sumado a los resultados de la intercalibración con el Laboratorio del ISTerre de la Universidad de Grenoble Alpes- Francia, de gran reputación y amplia experiencia a nivel internacional, demuestran que el Laboratorio de Termocronología del SGC cuenta con las capacidades técnicas, humanas y métodos estandarizados para desarrollar los análisis de huellas de fisión en apatitos y circones. Aunque el Zeta ( $\zeta)$, es un factor dinámico que en la práctica varía a medida que los observadores llevan a cabo nuevos análisis, los resultados obtenidos sugieren que todos los analistas del Laboratorio del SGC se encuentran dentro del rango de variación aceptable.

Los valores obtenidos durante los experimentos de irradiación de monitores de fluencia son muy favorables, estos indican que se pueden lograr las condiciones requeridas para la irradiación de muestras en tiempo razonables del orden de pocas horas y bajo las condiciones de flujo neutrónico requeridas. Por lo tanto, se recomienda avanzar en estudios para la caracterización del flujo neutrónico del Reactor Nuclear.

\section{AGRADECIMIENTOS}

Los autores agradecen al doctor Eduardo Posada del Centro Internacional de Física, Doctora Nidia Piragauta de Colciencias y al Doctor Armando Zamora de la Agencia Nacional de Hidrocarburos, quienes con su valiosa colaboración contribuyeron a que se lograra la implementación del Laboratorio de Termocronología del SGC.

\section{REFERENCIAS}

[1] M. I. Marín et al, Intercalibración de los laboratorios de Huellas de Fisión, Universidad EAFIT-JOSEPH FOURIER-INGEOMINAS, XIII Congreso Colombiano de Geología y XIV Congreso Latinoamericano de Geología, Medellín, Colombia. 2011.
[2] X. Bustos et al., Comparación de Superficies de Erosión en el Altiplano Antioqueño mediante Geomorfología Cuantitativa: Resultados preliminares. XIII Congreso Colombiano de Geología y XIV Congreso Latinoamericano de Geología, Medellín, Colombia. 2011.

[3] M. L. Peña, J. Sandoval y Y. Cañón, Laboratorios e Instalaciones de Tecnologías Nucleares y Reactor Nuclear del Servicio Geológico Colombiano, 14th Colombian Geological Congress, Bogotá, Colombia. 2013.

[4] L. Rayo et al., Resultados irradiaciones para análisis de huellas de fisión, reactor nuclear IAN-R1 del Servicio Geológico Colombiano, 14th Colombian Geological Congress, Bogotá, Colombia. 2013.

[5] A. Piraquive et al., Syntectonic erosion and exhumation processes in the Western Cordillera of Colombia, evidence from a tectonic stacking zone alongside the Romeral Suture Zone. 14th Colombian Geological Congress, Bogotá, Colombia. 2013.

[6] C. Urueña et al., Termocronología aplicada al estudio de evolución térmica en cuencas carboníferas (Sinclinal Umbita). XV Congreso Colombiano de Geología, Bucaramanga, Colombia. 2015a.

[7] C. Urueña et al., Termocronología aplicada en la Exploración de Gas Metano Asociado al Carbón. $\mathrm{X}$ Congreso Nacional y $\mathrm{V}$ Internacional de Ciencia y Tecnología del Carbón y Combustibles Alternativos - CONICCA 2015, Medellín, Colombia. 2015b.

[8] S. Amaya, C. Zuluaga and M. Bernet. Tectonic Evolution of the Northern Andes, Colombia: New Age Constrains on the Exhumation of the Santander Massif. Lithos, in review.

[9] S. Amaya, C. Zuluaga and M. Bernet. Three phases of accelerated exhumation along the Bucaramanga fault in the Cepita area of the south-western Santander Massif, Colombia, Tectonics, in review.

[10] R. L Fleischer, P. B. Priceand and R. M. Walker, Nuclear Tracks in Solids. Principles and 
Applications. University of California Press, Berkeley, CA. 1975.

[11] N. E. Holden, and D.C. Hofman, Spontaneous Fission Half-Lives for Ground-State Nuclides. Pure Appl. Chem, vol. 72, No. 8, pp. 1525-1562, () 2000 IUPAC. 2000.

[12] R. Donelick, P. B. O'Sullivan, and R. Ketcham, Apatite Fission-Track Analysis: Reviews in Mineralogy \& Geochemistry, v. 58, p. 49-94. 2005.

[13] G. A. Wagner and P. van den Haute, Fission-Track Dating. Netherlands: Solid Earth Sciences Library, Kluwer. Academic Publishers. 1992.

[14] K. Gallagher, R. Brown and C. Johnson, Fission track analysis and its applications to geological problems. Annual Review Earth Planetary Sciences, 26: 519-72. 1998

[15] A.J Hurford, and P. F. Green, A user's guide to fission track dating calibration. Earth Planetary Sciences Letters, 59: 343-354. 1982.

[16] A.J. Hurford, "Standardization of fission track dating calibration: Recommendation by the
Fission Track Working Group of Subcommision on Geochronology," Chemical Geology (Isotope Geoscience Section), vol. 80: 171 -178. 1990.

[17] P. W. Reiners and M. T. Brandon, "Using thermochronology to understand orogenic erosion," Annu. Rev. Earth. Planet. Sci., 34, 419 466. 2006.

[18] M. I. Marín et al., Calibración interlaboratorios de huellas de fisión. Universidad EAFIT - Servicio Geológico Colombiano - Universidad Joseph Fourier, 14th Colombian Geological Congress, Bogotá, Colombia. 2013.

[19] A.K Ghosh, V. K. Sharma and R. K. Singh, Uplift History of Syenite Rocks of the Sushina Hill, Tamar PorapaharShear Zone (TPSZ), Purulia: Constraints from Fission-track Ages of Two Cogenetic Minerals. Journal of Geology \& Geophysics 5: 245, doi:10.4172/2381-8719.1000245. 2016.

[20] M. L. Balestrieri, G. Bigazzi and M. Oddone, "The thermal column: a new irradiation position for fission-track dating in the University of Pavia Triga Mark II nuclear reactor," J. Radioanal. Nucl. Chem, Letters 213 (2):99-108. 1996 